\title{
Multilocus restriction typing: a tool for Neisseria meningitidis strain discrimination
}

\author{
Désirée E. Bennett ${ }^{1}$ and Mary T. Cafferkey ${ }^{1,2}$ \\ ${ }^{1}$ Epidemiology and Molecular Biology Unit, The Children's University Hospital, Temple Street, Dublin 1, \\ Ireland \\ ${ }^{2}$ Department of Clinical Microbiology, Royal College of Surgeons in Ireland, York Street, Dublin 2, \\ Ireland
}

Désirée E. Bennett

Desiree.Bennett@tsch.ie

\begin{abstract}
Invasive disease-associated strains of Neisseria meningitidis were analysed by multilocus restriction typing (MLRT), which involves the restriction fragment-length polymorphism analysis of PCR products generated from the seven loci of housekeeping genes used in MLST. Several different restriction patterns (alleles) were observed for each of the seven loci examined. Greater allelic variation was observed with the fum $C$ and $p g m$ loci than with the $a b c Z$ and adk loci, suggesting that the latter were more conserved. The alleles at each of the seven loci were combined to give an allelic profile or restriction type (RT). A good correlation between RT and serogroup, serotype and serosubtype was observed, as all C 2ap1.2,5 strains were contained in a single RT, as were all but one strain of B 4p1.4. In this study, MLRT proved to be an efficient, effective and relatively inexpensive method for $N$. meningitidis strain characterization.
\end{abstract}

Received 19 February 2003

Accepted 2 June 2003

\section{INTRODUCTION}

Neisseria meningitidis is a leading cause of invasive disease, including bacteraemia and meningitis, in both the developed and developing world. The molecular typing and subtyping of isolates of $N$. meningitidis has been the focus of much research over the past two decades (Cartwright \& Ala'Aldeen, 1997; Maiden \& Frosch, 2001; Yakubu et al., 1999). Several effective molecular techniques that examine isolates for characteristics that allow discrimination below the species level have been described (reviewed by Yakubu et al., 1999). Many of these have been adopted for routine strain characterization in the clinical setting, especially in the investigation of outbreaks and in determining the epidemiology of $N$. meningitidis. These have been used to provide information on isolate relatedness for both short- and long-term epidemiological purposes.

Multilocus enzyme electrophoresis (MLEE) and its recently developed DNA sequence-based counterpart, multilocus sequencing typing (MLST), are the most valuable techniques adapted for the study of the long-term or global epidemiology of N. meningitidis (Maiden et al., 1998; Selander et al., 1986). MLST offers many advantages, including speed and enhanced discrimination, over MLEE and has now almost entirely superseded MLEE as a strain typing method (Enright \& Spratt, 1999; Spratt, 1999; Yakubu et al., 1999). The

Abbreviations: MLRT, multilocus restriction typing; MLST, multilocus sequence typing; RT, restriction type. electronic portability of the nucleotide sequence data, enabling rapid global exchange of molecular typing information for epidemiological comparisons, is one of the biggest advantages of MLST over MLEE. However, while the technology for nucleotide sequencing has improved substantially in the last few years, with the advent of automated sequencers, sequencing of multiple housekeeping genes still remains time-consuming (Clarke et al., 2001a; Tondella et al., 1999). In addition, a further factor that must be taken into consideration with MLST is cost and, despite the additional commercial competition on the DNA sequencing market, automated DNA sequencing instruments remain expensive, as do fluorescently labelled dideoxynucleotide sequencing kits (Hookey \& Arnold, 2001; Goulding et al., 2000; Olive \& Bean, 1999). Initial start-up costs are high, ranging from approximately $\$ 100000$ to $\$ 300000$ (Diggle \& Clarke, 2002), and it is generally accepted that high throughput is required to minimize nucleotide sequencing costs. Therefore, to achieve maximum cost- and time-effectiveness and efficiency, an automated sequencer must be used to maximum capacity every time, which, in most laboratories, may correspond to a single use per week. Even in the recent description of semi-automated MLST performed using a robotic liquid-handling system, it was reported that MLST was probably only cost-effective in national reference laboratories or large clinical laboratories where high throughput could justify the cost (Clarke et al., 2001a). These authors later report that the cost of consumables alone for performing MLST on seven genes (for both strands) arrives at $\$ 32$ per bacterial isolate (Diggle \& Clarke, 2002). Further- 
more, manual DNA sequencing, even with an automated DNA sequencer, requires a high level of technical competence in carrying out the DNA sequencing reactions and interpreting the results and also in instrument maintenance. In short, in a standard laboratory without robotics and personnel dedicated to sequencing, it is too expensive, timeconsuming, laborious and tedious to perform complete MLST on seven loci for a large number of isolates, on a routine basis (Clarke et al., 2001a; Olive \& Bean, 1999; Shlush et al., 2002). A more user-friendly, relatively cheap, rapid and simple method based on the multilocus approach is required. A technique using the multilocus approach, based on cleavase fragment length polymorphism (CFLP) analysis of metabolic genes, was recently demonstrated to be reproducible, rapid and discriminatory for N. meningitidis epidemiology (Tondella et al., 1999). However, it has the drawback of being as complex and as expensive to perform as DNA sequencing (Olive \& Bean, 1999). A further technique with phylogenetic significance, fluorescent amplified-fragment length polymorphism (FAFLP) genotyping, has been applied successfully to identify hypervirulent, hyperendemic lineages of N. meningitidis (Goulding et al., 2000; Hookey \& Arnold, 2001). However, FAFLP analysis appears time-consuming and laborious and still requires the use of an automated sequencer. In a recent report, denaturing HPLC (DHPLC) was used as a simple and faster approach than DNA sequencing, for comparing the products obtained by MLST. The authors demonstrated $100 \%$ correlation between MLST in its original format and their DHPLC-MLST method (Shlush et al., 2002).

The goal of the studies described in this paper was to evaluate a simple, rapid and reproducible procedure as an alternative to MLST for multilocus typing of N. meningitidis isolates. An alternative to MLST is multilocus restriction typing (MLRT; Müller-Graf et al., 1999), in which variation at several loci is indexed by restriction fragment-length polymorphism (RFLP) analysis of PCR-amplified genes. During the preparation of the present report, a paper was published documenting the use of MLRT as a novel tool for studying the global epidemiology of Burkholderia cepacia (Coenye \& LiPuma, 2002). A further paper by the same researchers (Coenye et al., 2002) demonstrated a good agreement between MLRT and other commonly used genotyping methods for B. cepacia. Here, we describe MLRT analysis of the seven housekeeping genes used in MLST and, as an initial step in the assessment of MLRT, we compared data obtained by MLRT and serology for a large number of $N$. meningitidis isolates that were collected in Ireland.

\section{METHODS}

N. meningitidis isolates and strains. Eighty-three strains of $N$. meningitidis, whose serogroup, serotype and serosubtype were known, which included an ATCC serogroup C strain 13101 (Block, 2001) and three other reference strains, a serogroup A strain (M96/255449), a serogroup B strain (H44/76; Bjune et al., 1991) and a serogroup C strain (C11), were examined by MLRT analysis. Sixty-seven of these strains were recovered from either the blood or cerebrospinal fluid of 64 individuals with meningococcal disease and represented the most common serogroups, serotypes and serosubtypes associated with invasive meningococcal disease in Ireland. Two strains were recovered from each of three individuals, isolated from separate sites on different occasions. The remaining 12 strains were from individuals who did not have invasive meningococcal disease and were recovered from either throat, nasal or eye swabs. Of these 12 , three strains were recovered from separate individuals within the same family. All 79 clinical isolates were recovered in Ireland. DNA was extracted from all strains by using either one or both of two methods, the IsoQuick DNA extraction kit (Orca Research) or the microLYSIS extraction solution (Microzone).

Gene targets, PCR primers and cycling conditions. The seven gene targets $(a b c Z, a d k, a r o E, f u m C, g d h, p d h C$ and $p g m)$ and PCR primer pairs used in this study were those described previously for MLST analysis (Maiden et al., 1998). Touchdown PCR cycling conditions were used with annealing temperatures of $59^{\circ} \mathrm{C}$ for the initial three cycles, $57^{\circ} \mathrm{C}$ for the next three cycles followed by $52{ }^{\circ} \mathrm{C}$ for the final 30 cycles on a PTC 200 thermal cycler (MJ Research). These cycling conditions are slightly different from those described for MLST analysis by Clarke et al. (2001a). PCRs were performed in a final volume of $50 \mu \mathrm{l}$ consisting of $1 \mu \mathrm{l}$ extracted DNA as template, $5 \mu \mathrm{l} 10 \times$ PCR buffer, $1.25 \mathrm{U}$ Taq DNA polymerase (Gibco BRL) with 50 pmol of each primer and $0.2 \mathrm{mM}$ of each of dATP, dCTP, TTP and dGTP. A $\mathrm{MgCl}_{2}$ concentration of $2.5 \mathrm{mM}$ was used for all primer pairs except aroE primers, where $2.0 \mathrm{mM} \mathrm{MgCl}_{2}$ was used.

Restriction endonuclease typing. Following an initial study with a panel of 'frequent-cutting' restriction endonucleases ( $4 \mathrm{bp}$ recognition sequence) and a serogroup B and C strain, a single restriction endonuclease that yielded at least three fragments for each locus and strain was chosen. MspI was used to analyse PCR products of $a b c Z, a d k$, fum $C$ and $g d h$. MnlI was used to analyse the PCR products of $a r o E, p d h C$ and pgm. Restriction patterns were assessed on the basis of the number and size of restriction fragments obtained following digestion and electrophoretic separation $\left(4 \mathrm{~V} \mathrm{~cm}^{-1}\right)$ in a horizontal $3.5 \%(\mathrm{w} / \mathrm{v})$ agarose gel containing $0.5 \mu \mathrm{g}$ ethidium bromide $\mathrm{ml}^{-1}$. The restriction patterns obtained with each locus were analysed using the band detection and molecular mass determination features of the 1D Advanced software (version 4.01.2) and compared using the 1D Database software (version 1.12) available from Phoretix International to remove any subjectivity or bias in their analysis. Different allele numbers were assigned arbitrarily to patterns that differed in either the number and/or size of any of the bands. As with MLST, the combination of alleles at each of the seven loci gave an allelic profile or restriction type (RT) and strains were said to be different if the allele at any of the seven loci differed.

Using the RT data, specific clonal complexes within the 83 strains were investigated with the BURST algorithm within the START software package, version 1.0.0 (K. Jolley, University of Oxford, UK) (Jolley \& Urwin, 2001). In addition, a dendrogram was constructed using the percentage of alleles at which strains differed as the distance between them, as calculated using the unweighted pair group method with arithmetic mean (UPGMA) algorithm within the START software package.

\section{RESULTS}

\section{Restriction analysis of seven gene fragments}

Single PCR products of the same size for each of the seven genes analysed were obtained with each of the 83 strains examined. Restriction analysis of each of the seven loci produced between two and ten patterns consisting of between two and eight bands ranging from approximately 20 to 
880 bp, depending on the locus and strain examined (Fig. 1). The method used to extract the DNA from each strain did not appear to affect the amplification of any of the seven genes examined or the subsequent restriction analysis of the products obtained.

\section{MLRT of 83 strains}

Restriction patterns at each of the seven loci were combined to give an allelic profile or RT for each strain. Unique combinations of alleles yielded a new RT. From the 83 strains examined, 29 RTs were obtained and their relationships with each other are depicted in the dendrogram (Fig. 2). However, these must be interpreted with care, as RT6 is just as closely related to RT2 as it is to RT3, differing by just two loci, although this is not evident from the dendrogram. Five RTs (RT1, RT5, RT6, RT15 and RT16) contained $53(63.8 \%)$ of the strains, and RTs 2, 9, 11, 14, 17 and 21 contained two strains each. The remaining $18 \mathrm{RT}$ s each contained a single strain (Fig. 2). Each of the two strains recovered from the same individual yielded the same RT. No particular difference was noted between the RTs of disease-associated strains and carriage strains. Carriage strains were distributed throughout the dendrogram. Of the three carriage strains recovered from individuals within the same family, two yielded the same RT (RT5) and the other generated a unique RT (RT23) that differed at two loci from the RT of the other two strains.

Following analysis of the seven-loci RTs with the BURST program, the strains were grouped into two lineages, with a single singleton RT. Group 1 contained 26 RTs and 79 strains $(95 \cdot 2 \%)$ and group 2 contained only two RTs or three strains (RTs 21 and 24). RT7 was not represented in either of the groups, indicating that this RT varied by three or more loci from every other RT.

\section{MLRT association with serology}

The 83 strains examined included representatives of all the main serogroups of $N$. meningitidis, with 38 serogroup B strains, $30 \mathrm{C}$ strains, four each of W135 and NG, two each of $\mathrm{Y}$ and $\mathrm{X}$ and one each of A, 29E and Z (Fig. 2). Six RTs contained all the serogroup $\mathrm{C}$ strains examined. RTs 1 and 6 exclusively contained $25(83.3 \%)$ of these strains, all with either subtype $2 \mathrm{a}, \mathrm{NT}$ or $2 \mathrm{~b}$ and with serosubtype p1.2, p1.5, p1.2,5 or NT. The serogroup B strains examined yielded 14 different RTs. Eight RTs contained a single strain each and a further three RTs contained two strains each. A single RT, RT5, contained 14 serogroup B strains and, in conjunction with two other RTs, accounted for $24(63.5 \%)$ of all serogroup B strains. RT15 contained strains of serogroups $\mathrm{B}$ and $\mathrm{C}$, two strains of C $4 \mathrm{p} 1.10$ and three strains each of B 15p1.7,16 and B 4p1.15. This was the only RT to contain strains of both serogroups. Among the four serogroup W135 strains analysed, three RT combinations were obtained, which differed from each other by only either the fumC or pdhC locus. All of these were designated serotype NT and were either serosubtype p1.3,6 or NT. In addition, both serogroup X strains examined yielded the same RT, RT21, but both were of the same serology combination, X 21p1.16. (a) $a b c Z$

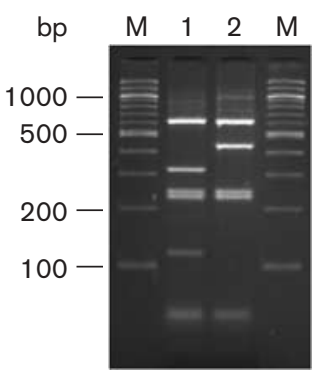

(e) $g d h$

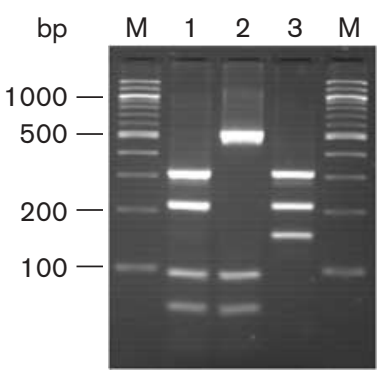

(b) $a d k$

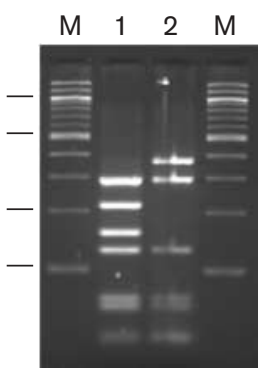

(c) $\operatorname{aro} E$

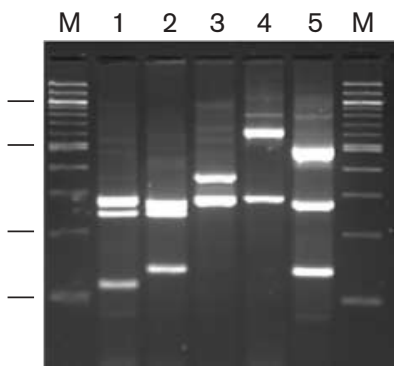

(d) fumC

$\begin{array}{lllllllllll}M & 1 & 2 & 3 & 4 & 5 & 6 & 7 & 8 & 9 & M\end{array}$

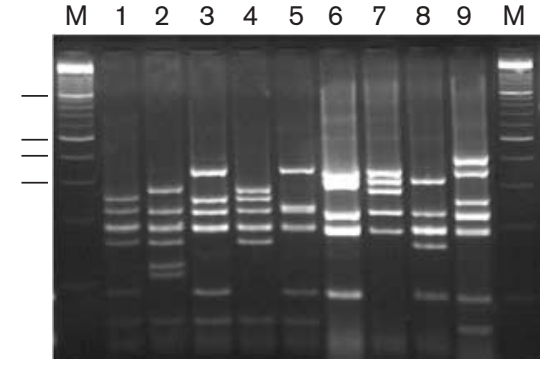

(f) $p d h C$

(g) pgm
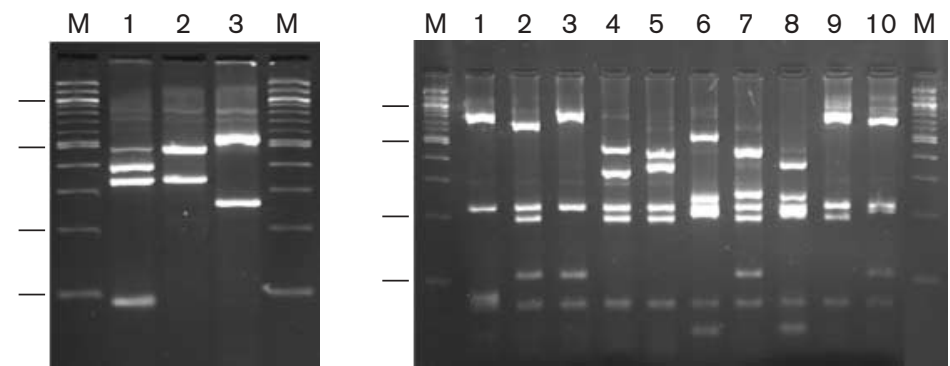

Fig. 1. Examples of different restriction patterns obtained following Mspl or Mnll digestion of PCR-amplified regions of the seven house-keeping genes analysed: $\operatorname{abcZ}(\mathrm{a}), \operatorname{adk}(\mathrm{b}), \operatorname{aro} E(\mathrm{c}), \operatorname{fum} C(\mathrm{~d}), \mathrm{gdh}(\mathrm{e}), \mathrm{pdhC}(\mathrm{f})$ and $\mathrm{pgm}(\mathrm{g})$. Lane numbers correspond to allele codes listed in Fig. 2. Lanes M, 100 bp ladder DNA size markers. 

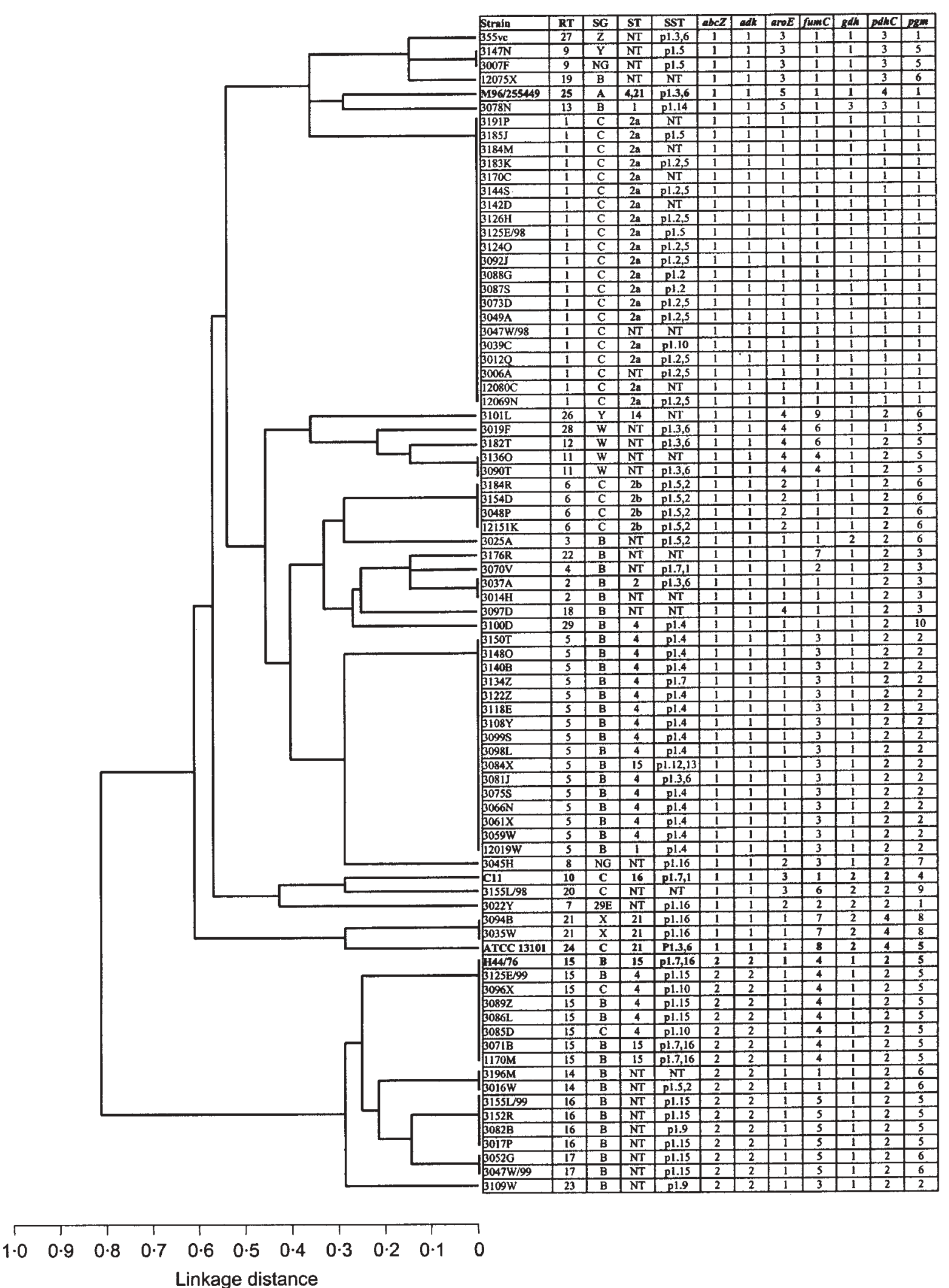

Fig. 2. Dendrogram showing the relatedness of 83 meningococcal strains based on analysis of their RT profiles generated by MLRT analysis, constructed using the UPGMA algorithm available in the START software package (Jolley \& Urwin, 2001). The RT profile, serological classification [serogroup (SG), serotype (ST) and serosubtype (SST)] and individual allele numbers for each locus (abcZ, adk, aroE, fumC, gdh, pdhC and pgm) for each strain are also included. 
These strains, along with the ATCC 13101 reference strain, formed the basis of the second lineage as defined by BURST analysis. The two serogroup Y strains examined yielded different RTs, differing at four loci, but were of completely different subtype and serosubtype combinations. Each of the strains of serogroups A, 29E and Z yielded unique RTs; the serogroup A strain was RT25, the serogroup 29E strain was RT7 and the serogroup Z strain was RT27. The four NG strains examined yielded three different RTs, RT5, 8 and 9. The serogroup 29E strain yielding RT7 constituted the only singleton as defined by BURST analysis. Strains of all other serogroups, A, B, C (except ATCC 13101), W135, Y, Z and $N G$, yielded RTs that were all deemed to be part of the same lineage as defined by BURST analysis.

In general, there was a good correlation between RT and serogroup, serotype and serosubtype, apart from single strain exceptions. Strains of RT1 $(n=21)$ were all serogroup C; 19 were serotype $2 \mathrm{a}$ and the serotypes of the remaining two were designated non-typable (NT) by the conventional serology typing scheme. Of these 21 strains, 10 were serosubtype p1.2,5, six were designated NT and the remaining five serosubtypes were p1.2, p1.5 (each $n=2$ ) and p1.10. All four C 2bp1.2,5 strains yielded RT6 and all but one of the $11 \mathrm{~B}$ 4 p1.4 strains examined yielded RT5. The remaining B 4p1.4 strain yielded a unique RT, RT29, differing in the fum $C$ and pgm alleles. However, RT5 also contained single B 4p1.7, B 1p1.4, B 4p1.3,6 and B 15p1.12,13 strains and two NG 4p1.4 strains. A further grouping of B NTp1.15 was also observed, with three of the five strains examined yielding the same RT, RT16. The other two B NTp1.15 strains were recovered from the same patient and differed at only one allele, the $p g m$ locus, from RT16, producing RT17 (Fig. 2). RT15 contained a variety of strains with three distinct serogroup, subtype and serosubtype combinations, three strains of B 15p1.7,16, three strains of B 4p1.15 and two strains of C 4p1.10.

Of the 12 strains recovered from individuals without invasive meningococcal disease, seven were contained in RTs along with strains having similar serology combinations, whereas the other five, each with unique serology combinations, yielded distinct RTs. It is difficult to make any further conclusions regarding congruence between RT and serological typing, as only single strains of other serology combinations or strains, whose specific serology combinations were unknown, as they were designated non-typable and nonserosubtypable by conventional serology, were examined. Among the four NG strains examined, three different RTs were obtained. The two NG 4p1.4 strains were contained in RT5 with strains of serogroup B possessing the same serotypes and serosubtypes. The NG NTp1.16 strain yielded a unique RT, RT8, and the NG NTp1.5 strains had the same RT, RT9, as a serogroup Y NTp1.5 strain.

\section{DISCUSSION}

In this study, 83 strains of $N$. meningitidis were characterized using MLRT, a DNA RFLP-based alternative to MLST, in an attempt to evaluate MLRT as a technique for N. meningitidis strain discrimination. This is the first description of MLRT as a technique for the discrimination of N. meningitidis, but it has been documented for the analysis of Streptococcus pneumoniae strains (Müller-Graf et al., 1999) and has recently been used for the study of the global epidemiology of B. cepacia (Coenye \& LiPuma, 2002; Coenye et al., 2002). MLRT divided the $83 \mathrm{~N}$. meningitidis strains into 29 different groups or RTs based on the combination of alleles or restriction patterns observed at each of the seven loci examined. Although discrimination is based on the analysis of banding patterns on agarose gels, patterns were easy to analyse and recognize because only between two and ten different patterns were observed for each locus, containing only a small number of bands (between two and eight) in each pattern. Greater allelic variation was observed with the pgm and fumC loci than with the $a b c Z$ and adk loci, suggesting that the latter were more conserved, features also observed with MLST (Maiden et al., 1998). Strain discrimination was based on all seven loci; however, analysis on just the aroE, fum $C, p d h C$ and $p g m$ loci would have divided these strains into the same RTs. The only situation in which this would not have discriminated between two RTs was with RT23 (represented by only one strain), which has the same pattern as RT5 at each of these four loci. This was not too surprising, as the three strains recovered from members of the same family were contained in these RTs. This indicates that these four loci were the most discriminatory, and a reduced MLRT analysis with these loci only would be sufficient to achieve the same discrimination.

Two clonal complexes and one singleton RT were identified following BURST analysis. The primary clonal complex contained all but three RTs, representing $95 \cdot 2 \%$ of the strains examined and including all the serogroup A, B, C (except ATCC 13101), W135, Y, NG and Z strains analysed. The second group contained the two serogroup $X$ strains and strain ATCC 13101, and the singleton RT was the serogroup $29 \mathrm{E}$ strain. To be included in a clonal complex/group, every strain must share at least five identical alleles with at least one other strain in that group. In the primary complex in this study, all 79 strains fulfil this criterion, but the five shared alleles are not the same five alleles in all of the 79 strains. Therefore, it is unlikely that these lineage delineations represent the true phylogenetic relationship among these strains.

An excellent association between RTs and serology was observed, apart from single strain exceptions. Strains of the same RT were mainly of the same serogroup and serotype combination, i.e. strains with serogroup $C$, serotype $2 \mathrm{~b}$ and serosubtype p1.2,5 were all contained within RT6, and strains with RT1 were predominantly serogroup C, serotype $2 \mathrm{a}$ and serosubtype p1.2,5. However, two strains whose serotypes were non-typable and six strains whose serosubtypes were non-typable were also contained within RT1. It is possible that these strains were $2 \mathrm{a}$ serotype and p1.2 or p1.5 or both serosubtypes but did not express the genes. Similarly, strains with RT5 were mainly B 4p1.4, although a B 4p1.4 strain also formed a unique RT, RT29, and RT5 also contained single B 
4p1.7, B 4p1.3,6, B 15p1.12,13 and B 1p1.4 strains. However, this is not surprising, as it has been documented previously that B 4p1.4 strains can be closely related genetically to strains of other diverse serological phenotypes (Van Looveren et al., 1998, 2001). Therefore, it could be concluded that serotype 4 could be important for inclusion in RT5, although other strains of serotype 4, three B 4p1.15 strains and two C 4p1.10 strains, yielded a different RT, RT15. RT15 also contained three strains with a B 15p1.7,16 phenotype. RT5 also contained two strains whose serogroup was nongroupable but whose serotypes were $4 \mathrm{p} 1.4$; this suggests that these strains were probably serogroup $B$ strains that did not express the B capsule protein. Furthermore, another NG strain, NG NTp1.5, yielded the same RT as a serogroup Y strain with a similar serology combination, Y NTp1.5. This could suggest that the NG strain was, in fact, a non-expressing serogroup $\mathrm{Y}$ strain.

In almost all cases, RTs contained strains of only one serogroup (apart from NG strains). Two RTs, RT1 and RT6, exclusively contained $83.3 \%$ of serogroup C strains examined and were either subtype $2 \mathrm{a}$, NT or $2 \mathrm{~b}$ with serosubtype p1.2, p1.5, NT or p1.2,5. These serotypes and serosubtypes represent $98 \%$ of the types and subtypes of the serogroup C isolates recovered in Ireland during 2000 and approximately $88 \%$ of serogroup C isolates recovered during 1999. Serogroup C strains were contained in only four other RTs. This suggests that the prevalent serogroup C strains associated with invasive meningococcal disease in Ireland are a genetically homogeneous population. In contrast, 14 RTs contained all the serogroup B strains examined. RTs 5, 15 and 16 contained $63 \%$ of all serogroup B strains examined. These strains were predominantly subtypes 4 , NT and 15 and serosubtypes p1.4, p1.7, p1.15, NT and p1.7,16. These serotype and serosubtype combinations account for approximately $65 \%$ of all serogroup B isolates recovered during 1999 and 2000 in Ireland. RT15 contained the Norwegian serogroup B strain, H44/76, which has previously been designated as belonging to the clonal complex ST-32 complex/ ET-5 complex by MLST/MLEE characterization (http:// www.mlst.net).

In this study, there was no evidence of strains with the same genetic background possessing different serogroups, suggestive of capsular switching, although there was evidence of poor or absent capsule expression. However, any serogroup B isolates yielding RT1 or RT6 or serogroup C isolates yielding RT5 could be indicative of capsular switching. The phenomenon of capsule switching has been described by a number of groups (Swartley et al., 1997; Vogel et al., 2000) and, in light of the recent introduction of MenC vaccination in Ireland (October 2000), it is important to monitor strains for capsular switching. The significance of RT15 containing both serogroup B and C strains is unknown, except that strains of both serogroups have been previously documented to belong to the clonal complex ST-32 complex/ET-5 complex, based on a search of the MLST database (http:// www.mlst.net), although none with the $\mathrm{C} 4 \mathrm{p} 1.10$ serotype and serosubtype combination was documented.
MLRT is an application of proven concepts and a variation on the methods of MLST and is therefore suitable for longterm or global epidemiological purposes. Many of the advantages associated with MLST are also valid for MLRT, including $100 \%$ typability, enhanced discrimination achievable through using a multilocus approach and potential for typing organisms directly from clinical material (Clarke et al., 2001b; Kriz et al., 2002). Other advantages of MLRT are simplicity, speed and relative cost and, from this limited study, it can be seen that MLRT also affords a high degree of discrimination, yielding an excellent congruence with serology.

The power of discrimination possible by MLST is undoubtedly much greater than could be expected from MLRT, even though larger gene fragments are examined in MLRT than are sequenced in MLST. Additional major advantages of MLST over MLRT include the portability of sequence data versus restriction patterns and the unambiguity of allele identity in cases where fragments with the same electrophoretic mobility may have completely different sequences. Nevertheless, the benefits of MLRT, combining high discriminatory power and acceptable turnaround time and lower cost compared with complete MLST analysis (Hookey \& Arnold, 2001; Olive \& Bean, 1999; Shlush et al., 2002), outweigh these advantages, especially for an 'in-house' initial screening method. MLRT is easy to perform, without the need for a high level of technical expertise or the use of expensive specialized equipment; therefore, it could be performed on relatively large numbers of isolates in a routine molecular laboratory. However, as the number of isolates to be examined increases, potentially yielding several distinct DNA banding patterns that may be difficult to compare visually, computer-assisted image acquisition and DNA pattern analysis software may be required, at least to eliminate user bias and subjectivity in restriction pattern classification. An additional benefit of MLRT is its potential for adaptability to MLST, as the previously generated PCR product can be used as a sequencing template. It is these advantages that point towards the use of MLRT as an initial screening method to distinguish different isolates at a basal level without the need for sequencing. Isolates that are indistinguishable by MLRT can then be typed further by MLST without the need to repeat the PCR.

To conclude, in this study, MLRT has proved to be a discriminatory, reproducible, easy-to-perform, rapid and relatively inexpensive typing method to characterize $N$. meningitidis isolates genetically in a way that reflects their serological classification. However, the reliance of MLRT on comparisons of DNA patterns indicates its use either as an 'in-house' alternative to MLST and/or as an initial screening method to distinguish different isolates prior to MLST analysis. We expect that MLRT will be of value during epidemiological investigations of potential meningococcal outbreak situations, especially in laboratories that do not have direct access to robotics, automated sequencing facilities and specifically trained personnel. 


\section{ACKNOWLEDGEMENTS}

This work was presented in part at the Twelfth International Pathogenic Neisseria Conference, 12-17 November 2000, Galveston, TX, USA. We gratefully acknowledge Charles O'Neill for his suggestions and help in preparing this manuscript. We thank Edward J. Feil (Department of Biology and Biochemistry, University of Bath, UK) for his technical assistance with BURST and dendrogram analysis. We also thank Steve Gray (Manchester Public Health Laboratory Services, Withington Hospital, Manchester, UK) for providing the reference strains H44/ 76, C11 and the serogroup A strain M96/255449.

\section{REFERENCES}

Bjune, G., Høiby, E. A., Grønnesby, J. K. \& 15 other authors (1991). Effect of outer membrane vesicle vaccine against group B meningococcal disease in Norway. Lancet 338, 1093-1096.

Block, C. (2001). Antibiotic susceptibility testing. Methods Mol Med 67, 89-106.

Cartwright, K. A. \& Ala'Aldeen, D. A. (1997). Neisseria meningitidis: clinical aspects. J Infect 34, 15-19.

Clarke, S. C., Diggle, M. A. \& Edwards, G. F. (2001a). Semiautomation of multilocus sequence typing for the characterization of clinical isolates of Neisseria meningitidis. J Clin Microbiol 39, 3066-3071.

Clarke, S. C., Diggle, M. A. \& Edwards, G. F. (2001b). Automated nonculture-based sequence typing of meningococci from body fluids. $\mathrm{Br} J$ Biomed Sci 58, 230-234.

Coenye, T. \& LiPuma, J. J. (2002). Multilocus restriction typing: a novel tool for studying global epidemiology of Burkholderia cepacia complex infection in cystic fibrosis. J Infect Dis 185, 1454-1462.

Coenye, T., Spilker, T., Martin, A. \& LiPuma, J. J. (2002). Comparative assessment of genotyping methods for epidemiologic study of Burkholderia cepacia genomovar III. J Clin Microbiol 40, 3300-3307.

Diggle, M. A. \& Clarke, S. C. (2002). What a load of old sequence!!! J Clin Microbiol 40, 2707.

Enright, M. C. \& Spratt, B. G. (1999). Multilocus sequencing typing. Trends Microbiol 7, 482-487.

Goulding, J. N., Hookey, J. V., Stanley, J., Olver, W., Neal, K. R., Ala'Aldeen, D. A. \& Arnold, C. (2000). Fluorescent amplified-fragment length polymorphism genotyping of Neisseria meningitidis identifies clones associated with invasive disease. J Clin Microbiol 38, 4580-4585.

Hookey, J. V. \& Arnold, C. (2001). A comparison of multilocus sequence typing and fluorescent fragment-length polymorphism analysis genotyping of clone complex and other strains of Neisseria meningitidis. J Med Microbiol 50, 991-995.

Jolley, K. \& Urwin, R. (2001). Computational methods for meningococcal population studies. Methods Mol Med 67, 357-373.
Kriz, P., Kalmusova, J. \& Felsberg, J. (2002). Multilocus sequence typing of Neisseria meningitidis directly from cerebrospinal fluid. Epidemiol Infect 128, 157-160.

Maiden, M. C. \& Frosch, M. (2001). Molecular techniques for the investigation of meningococcal disease epidemiology. Mol Biotechnol 18, 119-134.

Maiden, M. C., Bygraves, J. A., Feil, E. \& 10 other authors (1998). Multilocus sequence typing: a portable approach to the identification of clones within populations of pathogenic microorganisms. Proc Natl Acad Sci U S A 95, 3140-3145.

Müller-Graf, C. D. M., Whatmore, A. M., King, S. J. \& 7 other authors (1999). Population biology of Streptococcus pneumoniae isolated from oropharyngeal carriage and invasive disease. Microbiology 145, 3283-3293.

Olive, D. M. \& Bean, P. (1999). Principles and applications of methods for DNA-based typing of microbial organisms. J Clin Microbiol 37, 1661-1669.

Selander, R. K., Caugant, D. A., Ochman, H., Musser, J. M., Gilmour, M. N. \& Whittam, T. S. (1986). Methods of multilocus enzyme electrophoresis for bacterial population genetics and systematics. Appl Environ Microbiol 51, 873-884.

Shlush, L. I., Behar, D. M., Zelazny, A., Keller, N., Lupski, J. R., Beaudet, A. L. \& Bercovich, D. (2002). Molecular epidemiological analysis of the changing nature of a meningococcal outbreak following a vaccination campaign. J Clin Microbiol 40, 3565-3571.

Spratt, B. G. (1999). Multilocus sequence typing: molecular typing of bacterial pathogens in an era of rapid DNA sequencing and the Internet. Curr Opin Microbiol 2, 312-316.

Swartley, J. S., Marfin, A. A., Edupuganti, S., Liu, L.-J., Cieslak, P., Perkins, B., Wenger, J. D. \& Stephens, D. S. (1997). Capsule switching of Neisseria meningitidis. Proc Natl Acad Sci U S A 94, 271-276.

Tondella, M. L., Reeves, M. V., Popovic, T., Rosenstein, N., Holloway, B. P. \& Mayer, L. W. (1999). Cleavase fragment length polymorphism analysis of Neisseria meningitidis basic metabolic genes. J Clin Microbiol 37, 2402-2407.

Van Looveren, M., Carion, F., Vandamme, P. \& Goossens, H. (1998). Surveillance of meningococcal infections in Belgium. Clin Microbiol Infect 4, 224-228.

Van Looveren, M., Caugant, D. A., Chapelle, S., Carion, F. \& Goossens, H. (2001). Interpreting the rising incidence of meningococcal disease in Belgium: the contribution of molecular typing. J Med Microbiol 50, 986-990.

Vogel, U., Claus, H. \& Frosch, M. (2000). Rapid serogroup switching in Neisseria meningitidis. N Engl J Med 342, 219-220.

Yakubu, D. E., Abadi, F. J. \& Pennington, T. H. (1999). Molecular typing methods for Neisseria meningitidis. J Med Microbiol 48, 1055-1064. 